\title{
Small-Strip Thin Gap Chambers for the Muon Spectrometer Upgrade of the ATLAS Experiment
}

\author{
D. Pudzha, ${ }^{a}$ on behalf of the ATLAS Muon Collaboration \\ ${ }^{a} N R C$ «Kurchatov Institute» - PNPI, \\ 188300, Leningradskaya oblast Gatchina1, mkr. Orlova roshcha, Russia \\ E-mail: dennis.pudzha@cern.ch
}

\begin{abstract}
The instantaneous luminosity of the Large Hadron Collider at CERN will be increased by about a factor of five with respect to the design value by undergoing an extensive upgrade program over the coming decade. In order to cope with the increasing luminosity, the ATLAS experiment is being upgraded as well. The largest phase-1 upgrade project for the ATLAS Muon System is the replacement of the present first station in the forward regions with the New Small Wheels (NSWs) during the long-LHC shutdown in 2019-2021. The NSWs consist of two detector technologies: Micromegas (MM) and small-strip TGC (sTGC), the later arranged in 2 quadruplet, each consisting of 8 layers. To retain the good precision tracking and trigger capabilities in the high background environment of the high luminosity LHC, each sTGC plane must achieve a spatial resolution better than $100 \mu \mathrm{m}$. It will allow to reconstruct tracks with an angular resolution of approximately $1 \mathrm{mrad}$. The sTGC design, performance, construction and integration status are discussed, along with results from tests of the chambers with nearly final electronics with beams, cosmic rays and high-intensity radiation sources.
\end{abstract}

KeYwords: Particle tracking detectors (Gaseous detectors), Muon spectrometers, Wire chambers (MWPC, Thin-gap chambers, drift chambers, drift tubes, proportional chambers etc)

\footnotetext{
${ }^{\text {a }}$ Speaker.
} 


\section{Contents}

1 Introduction 1

2 Motivation for the upgrade 1

3 sTGC for the NSW 2

4 STGC construction $\quad 3$

5 Performance of the sTGC 5

6 Conclusion 6

\section{Introduction}

The Large Hadron Collider (LHC) complex will be upgraded in several phases. The upgrade of the LHC will result in a factor of 5 more integrated luminosity with respect to the design value which will allow the reach of the physics program to be significantly extended.

To prepare the ATLAS experiment [1] for these new running conditions with a minimum impact on the physics performance, parts of the muon end-cap inner stations will be replaced by an assembly of the new detector modules called the New Small Wheels (NSWs) [2]. The NSW is designed to allow reconstructing of muon tracks with high precision and provide precise information for the Level-1 (L1) trigger using small-strip thin gap chambers (sTGC) [3, 4] and the Micromegas (MM) [5].

\section{Motivation for the upgrade}

The Level-1 muon trigger in the end-cap region is based on track segments in the TGC chambers of the middle muon station (End-cap Muon detector, EM) located after the end-cap toroid magnet. The transverse momentum, $p_{\mathrm{T}}$, of the muon is determined by the angle of the segment with respect to the direction pointing to the interaction point. A significant part of the muon trigger rate in the end-caps is background. Low energy particles, mainly protons, generated in the material located between the Small Wheel (SW) and the EM station, produce fake triggers by hitting the end-cap trigger chambers at an angle similar to that of real high $p_{\mathrm{T}}$ muons. As shown in Figure 1, a large fraction of the single-muon L1 triggers currently originate from the forward regions and approximately $90 \%$ of those triggers could not be matched to a $p_{\mathrm{T}}>10 \mathrm{GeV}$ muon after offline reconstruction. As a consequence the rate of the Level-1 muon trigger in the end-cap is eight to nine times higher than that in the barrel region.

In order to reduce the number of fake triggers the current SWs will be replaced with the NSWs which will be included in the trigger chain with the Big Wheels (BWs) allowing to exclude low $p_{\mathrm{T}}$ 


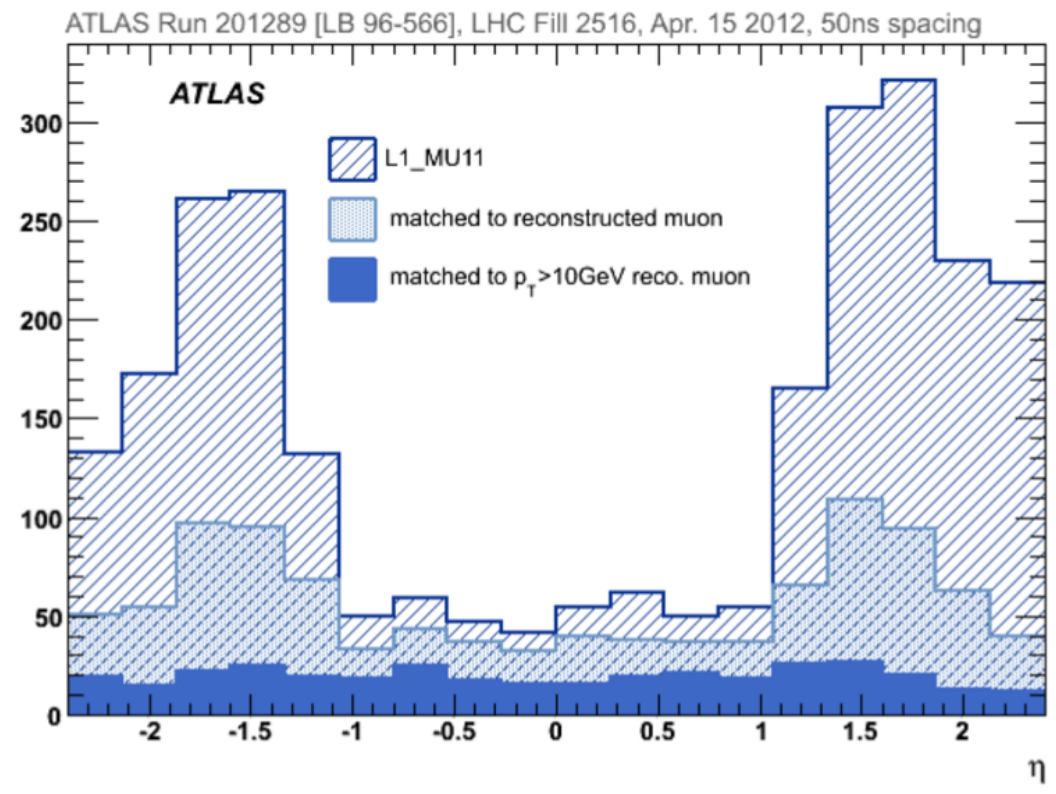

Figure 1. Distribution of the number of single-muon L1 triggers identified with $p_{\mathrm{T}}>10 \mathrm{GeV}$ as a function of pseudorapidity compared to the number of those triggers which are associated to a muon reconstructed offline [2]. CCopyright 2020 CERN for the benefit of the ATLAS Collaboration. CC-BY-4.0 license.

particles (mainly neutrons and photons) spontaneously exited from the endcap toroid. This leads to strict requirements for the NSW technologies.

Track segment reconstruction for triggering should have an angular resolution of $1 \mathrm{mrad}$ (RMS) or better to ensure sufficient reduction of the fake trigger rate. Other relevant detector performance criteria consists of the time jitter of detector hits which must be better than $25 \mathrm{~ns}$ for accurate bunch crossing identification. The spatial resolution in the bending plane should be better than $50 \mu \mathrm{m}$, to match the performance of the current MDT system. This will ensure that the muon spectrometer has a momentum resolution better than $10 \%$ at $p_{\mathrm{T}} \sim 1 \mathrm{TeV}$. This performance should not degrade even if a considerable fraction of the detected hits are caused by background particles or if some detector planes are not operational. Therefore, the required segment position resolution is better than $100 \mu \mathrm{m}$ resolution per plane for the planned 4-layer multi-plane detector.

\section{3 sTGC for the NSW}

The detector technologies that satisfy all aforementioned requirement and that were chosen for the NSW are sTGCs and MMs. As shown in Figure 2 the NSW combines eight layers of MMs mounted between four layers of sTGC on each side. The detectors are assembled into trapezoidshaped modules of four layers called quadruplets. Quadruplets are then mounted into pie-slice "wedges" consisting of 3 quadruplets for sTGC or 2 for Micromegas. Two types of wedges - large and small ones - make up the NSW.

sTGCs are multiwire proportional chambers with relatively thin gas volume with an anodecathode spacing of $1.4 \mathrm{~mm}$. The spacing between the anode wires, which are made of gold-plated tungsten, is $1.8 \mathrm{~mm}$. The cathode planes are formed out of several layers. At their base, they are 


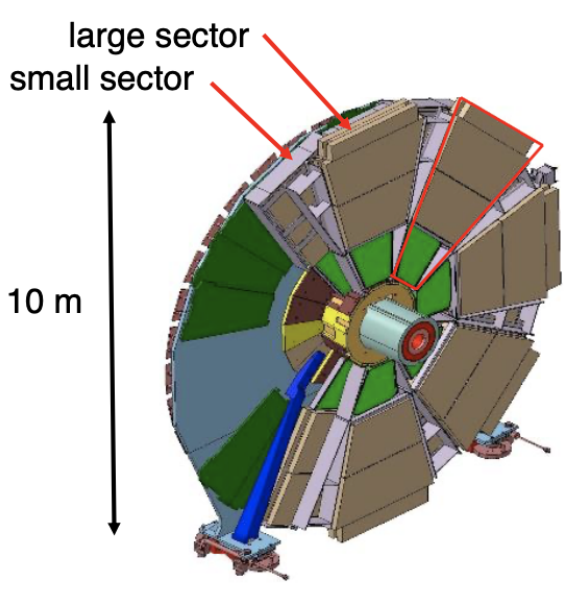

(a)

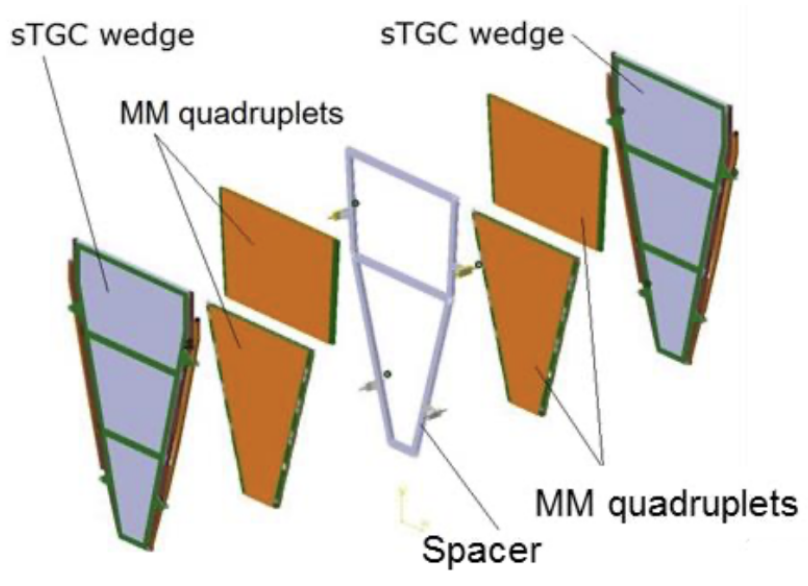

(b)

Figure 2. (a) Cutaway drawing of the finished NSW as seen looking towards the interaction point and (b) schematic of a disassembled large sector combining sTGC and Micromegas wedges. The sTGC wedges combine 3 modules while Micromegas wedges have 2 modules. The detector modules are made of 4 detector layers. Wedges can be of "large" or "small" type making large and small sectors respectively. The NSW is made of 8 large and 8 small sectors.

(C) Copyright 2020 CERN for the benefit of the ATLAS Collaboration. CC-BY-4.0 license.

a $1.1-1.3 \mathrm{~mm}$ thick PCB with a $100-200 \mu \mathrm{m}$ thick layer of pre-preg over top of them. Over this, the cathode planes are sprayed with a mixture of graphite and epoxy. One of the cathode planes is segmented into rectangular pads that are used in the trigger system to identify regions of interest in the strips and wires. The other cathode plane is segmented into $3.2 \mathrm{~mm}$ pitch strips in the azimuthal direction. The chambers are operating in the quasi-saturated mode at $2.85 \mathrm{kV}$ with a gas amplification of $2 \cdot 10^{5}$, which, combined with an average of 25 clusters ( 2 electrons each) produced by the passage of a minimum ionizing particle in the gas mixture of $\mathrm{CO} 2 / \mathrm{n}$-pentane, provides a total charge of $1.5 \mathrm{pC}$ (MPV), with $20 \%$ of the charge in the first 25ns. A schematic of a sTGC gas volume showing the different electrode types is shown in Figure 3. Individual sTGC layers are then assembled into modules consisting of four sTGC layers, known as quadruplets.

Manufactured modules are large, with surface areas varying between 0.7 and $2.3 \mathrm{~m}^{2}$, while requiring very precise positioning of readout elements. Therefore, every step of the assembly must follow a stringent quality control procedure whereby parts with unacceptable non-conformities in planarity, thickness or positioning of readout elements are rejected. A precise dimensional control of readout elements is also performed such that deviations of detector features with respect to nominal are known within $40 \mu \mathrm{m}$ in the radial coordinate and $80 \mu \mathrm{m}$ along the beam pipe.

\section{4 sTGC construction}

sTGC quadruplets for the NSW are produced in Russia, Israel, Canada, China and Chile. Three quadruplets are assembled into wedges at CERN. 


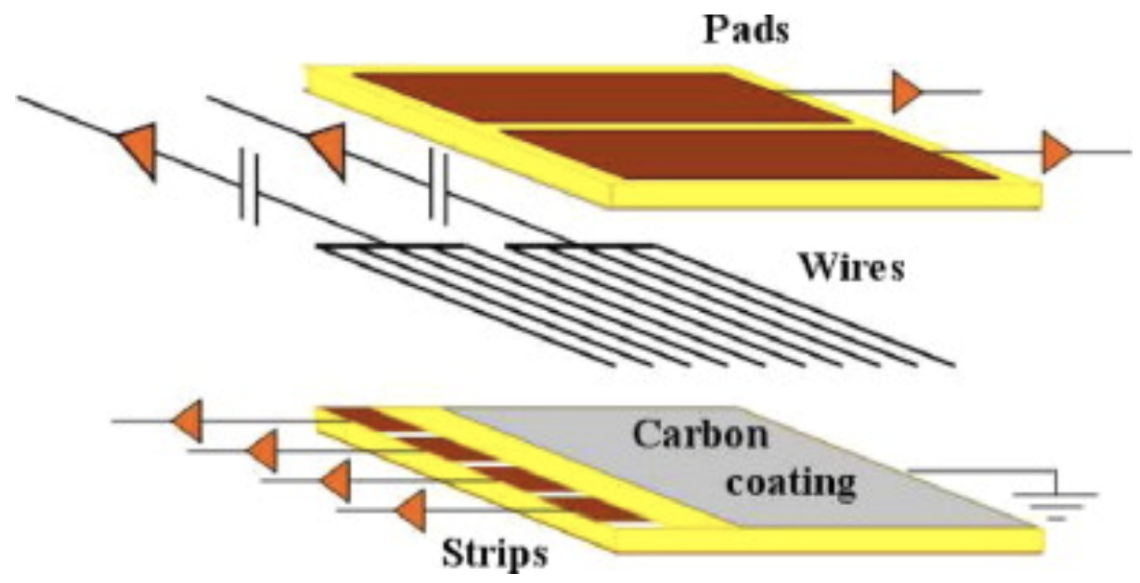

Figure 3. Schematic of the pad, strip and wire electrodes of a sTGC gas volume used in the NSW. The wires are ganged in groups of 20 and capacitively coupled to the readout electronics. The strip and pad electrodes are capacitively coupled to the graphite coating.

CCopyright 2020 CERN for the benefit of the ATLAS Collaboration. CC-BY-4.0 license.

As it was mention in section 3, chambers must be build with very high precision. The basic philosophy in the construction procedure of the quadruplets, is to produce a high precision strip board that can be referenced from the outside for each plane and for each quadruplet. This is achieved by placing brass inserts in the strip boards that are machined together with the strips. These inserts protrude outside the detector volume and can always be positioned with respect to precision pins on a flat granite table. The precision is obtained in the single detector level. Such a structure has the needed stiffness to be pressed against the precision pins. The requirement of $70 \mu \mathrm{m}$ maximal deviation between planes is hard to achieve due to the non-uniformity of the FR4 boards. This is alleviated by using paper-honeycomb fillers $40 \mu \mathrm{m}$ thinner than their corresponding frames, which permits local deformations of up to $30 \mu \mathrm{m}$ to be filled by the glue, while the cathodes are always attached to a flat surface through vacuum. The assembly of a quadruplet is made of two doublets; where each doublet is constructed as a symmetric structure, with the pad cathode board, that carry the wire planes, being always in the external side.

As part of the quality control procedure, HV tests are performed at each stage (single gaps, doublets, quadruplets) to identify shorts, sparks and leakage currents. To ensure gain uniformity and locate hot spots, gas volumes are scanned with X-rays. The gain should be uniform within $20 \%$ excluding wire supports and buttons.The connectivity of the signal lines is checked with a pulser test which consists of injecting pulses in the module high-voltage line while reading out the signal induced at the level of the signal lines. Finally, modules are tested with cosmic rays to check for noise level, hit rate and efficiency at nominal operation mode with single-like particles. A quadruplet is considered acceptable if each of its planes has an efficiency exceeding $95 \%$ on $95 \%$ of its sensitive area. Upon reception at CERN, modules are tested for the stability under high radiation conditions at the GIF++ irradiation facility [6]. Assembled wedges are placed in an isolated room where they are flushed with the $\mathrm{CO} 2 / \mathrm{n}$-pentane mixture and have $2.8 \mathrm{kV}$ nominal voltage applied 
onto them for a period of 2 months to ensure long-term stability.

The position of sTGC wedges in the ATLAS coordinate system is measured with an optical system that combines reference platforms holding light fibres and CCD cameras attached to the NSW frame. The position of cathode strips with respect to the alignment platforms is obtained from an X-ray scan performed after wedge assembly.

\section{Performance of the sTGC}

The charge response was measured using production sTGC modules during beam test campaigns at CERN. The collected charge distribution for different values of applied high-voltage for the QS3 module, the biggest type of the sTGC chamber in small sector of the NSW, using a low-rate muon beam in the H8 beam-test area, is shown in Figure 4(a). The noise pedestal distribution for the same pad is shown as well for comparison. The shapes follow a Landau distribution and, at the nominal operation voltage of $2.8-2.9 \mathrm{kV}$, sufficient separation from the noise pedestal can be seen. The normalised sTGC pad charge distribution for different background rates for the QL1 module, the smallest type of the sTGC chamber in large sector of the NSW, as measured in GIF++ using a muon beam in the presence of high rate photon background in GIF++, is shown in Figure 4(b). All distribution shapes follow a Landau distribution and, at a voltage of $2.8 \mathrm{kV}$, sufficient separation from the noise pedestal can be seen for all photon rates below $10 \mathrm{kHz} / \mathrm{cm}^{2}$, the target for ATLAS operations. The front end electronics and readout used belong to an intermediate demonstrator version and hence small improvements can be expected for the respective final versions.

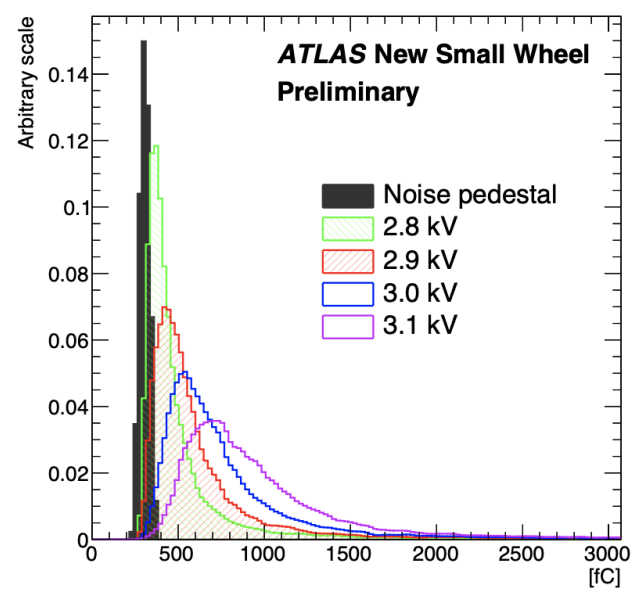

(a)

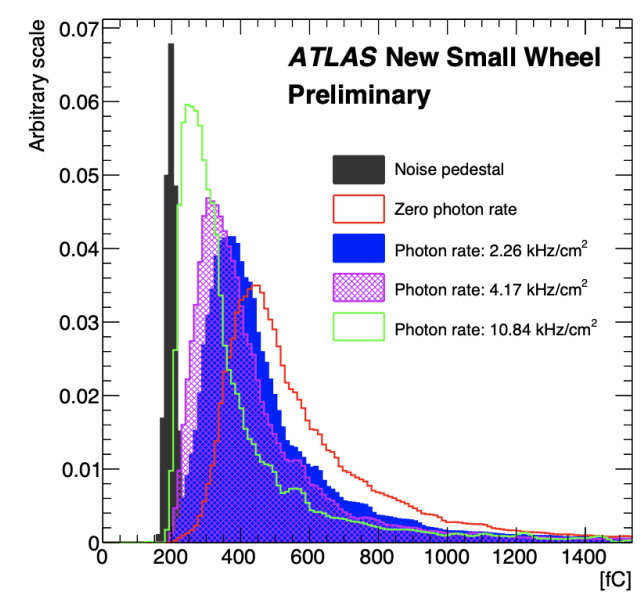

(b)

Figure 4. Charge distribution from a sTGC pad at (a) the H8 beam line for the QS3 module and at (b) the GIF++ facility for the QL1 module [7].

CCopyright 2020 CERN for the benefit of the ATLAS Collaboration. CC-BY-4.0 license.

The strip spatial resolution was also measured at the CERN H8 beamline using a production module. The strip spatial resolution is obtained from the distributions of the exclusive and inclusive residuals of the reconstructed tracks. The muon position was obtained using the centroid position 
of the charge profile of strips above threshold. The effect of differential non-linearity is corrected for during the offline analysis. All correction parameters are obtained in-situ using beam data. As shown in Figure 5, a spatial resolution better than $100 \mu \mathrm{m}$ was measured at the nominal high voltage of $2.8 \mathrm{kV}$ which indicates adequate performance to maintain the current $p_{\mathrm{T}}$ resolution of the muon spectrometer. The presented resolutions were obtained without using the "near-neighbour logic", which allows to set a higher threshold with respect to the noise (in particular important for low operating voltages), while keeping a good multiplicity to find the charge centroid [8].

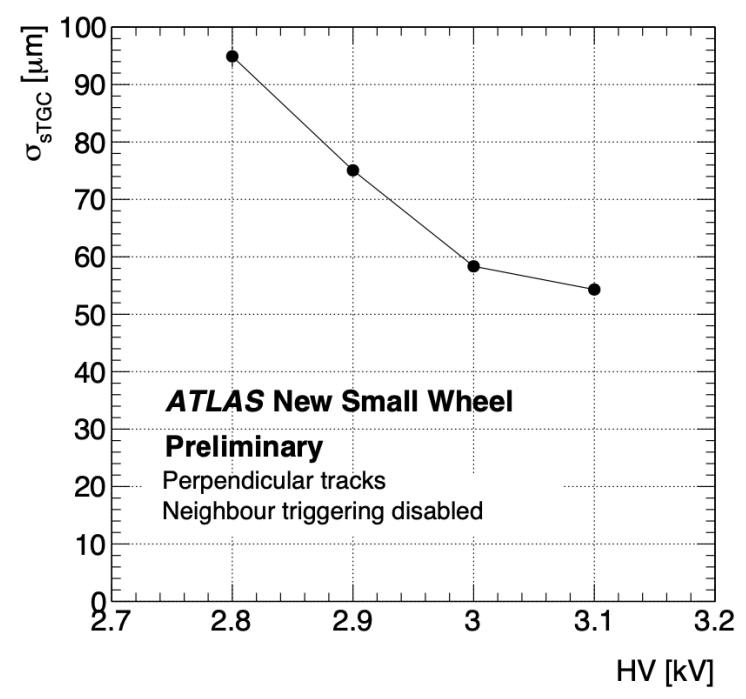

Figure 5. Intrinsic strip spatial resolution measured at the $\mathrm{H} 8$ beam line, without the near-neighbour logic [7]. (C) Copyright 2020 CERN for the benefit of the ATLAS Collaboration. CC-BY-4.0 license.

\section{Conclusion}

The NSW is necessary for the upgrade of the ATLAS muon system to achieve high trigger efficiency and high rejection of fake muons at the high radiation environment expected for high luminosity running at LHC. The design of front-end electronics was finalized following intense optimization campaigns and have been proven to fulfil the requirements. The NSW structure and services, which include high voltage cables, cables for digital communication, cooling and gas pipes, are ready for sector integration. The QA/QC procedure was shown to be useful in order to meet the requirements and the sTGC are able to achieve needed precision.

\section{References}

[1] ATLAS Collaboration,, The ATLAS experiment at the CERN Large Hadron Collider, J.Instrum 3 (2008) S08003.

[2] T. Kawamoto et al., New Small Wheel Technical Design Report, CERN-LHCC-2013-006. ATLAS-TDR-020, Jun, 2013. 
[3] G. Bella et al., Development of Calorimeters Using Thin Chambers Operating in a High Gain Mode, Nucl.Instrum.Meth.A 252 (1986) 503-510, Nucl. Instrum. Methods A252 ( 1986) 503-510.

[4] G.Mikenberg, Thin-gap gas chambers for hadronic calorimetry, Nucl.Instrum.Meth.A 265 (1988) 223-227.

[5] Y. Giomataris, P. Rebourgeard, J. Robert and G. Charpak, Micromegas: a high-granularity position-sensitive gaseous detector for high particle-flux environments, Nuclear Instruments and Methods in Physics Research Section A 376 (1996) 29.

[6] S. Agosteo et al., A facility for the test of large-area muon chambers at high rates, Nucl. Instrum. Methods Phys. Res. A 452 (2000) 94.

[7] New Small Wheel Public Results, https://twiki.cern.ch/twiki/bin/view/AtlasPublic/NSWPublicResults\#sTGC_Performance. Accessed: 2020-05-14.

[8] A. Abusleme et al., Performance of a full-size small-strip thin gap chamber prototype for the ATLAS new small wheel muon upgrade, Nucl. Instrum. Methods Phys. Res. A 817 (2016) 85. 